Journal of Elasticity, Vol. 5, Nos. 3-4, November 1975

Dedicated to A. E. Green

Noordhoff International Publishing - Leyden

Printed in The Netherlands

\title{
The angular dislocation in a half space
}

\section{MARIA COMNINOU}

Department of Applied Mechanics and Engineering Science, University of Michigan, Ann Arbor, Michigan 48104, USA

J. DUNDURS

Department of Civil Engineering, Northwestern University, Evanston, Illinois 60201, USA

(Received February 24, 1975)

\begin{abstract}
The solution for an angular dislocation allows one to construct the fields for any polygonal loop by superposition. The paper presents the displacements induced by the angular dislocation in an elastic half space. In view of potential applications in geophysics, particular attention is paid to the elastic fields at the free surface. The surface data are seen to exhibit a very simple dependence on the elastic constants.
\end{abstract}

\section{RÉSUMÉ}

On peut construire les champs élastiques associés à une dislocation en polygone par superposition de solutions au problème d'une dislocation angulaire. Nous présentons les déplacements induits par une dislocation angulaire dans un demi-éspace élastique. En vue des applications géophysiques, les champs élastiques sur la surface libre sont étudiés en particulier. Nous montrons que les champs élastiques sur la surface dépendent des constantes élastiques d'une façon très simple.

\section{Introduction}

The classical concept of a Volterra dislocation involves two requirements. The first is that the cut not terminate inside the material. The body must therefore be multiply connected, and the cut has to reduce its connectivity at least by one. The second requirement is that the relative motion of one side of the cut with respect to the other, or the discontinuity in the displacement field is of the same form in position coordinates as the infinitesimal motion of a rigid body. The consequences of these conditions are that the mechanical operation of dislocating induces strain that is continuous at the cut. Whereas traction must be continuous at the cut on account of Newton's third law, continuity of strain in the elastic body implies continuity of all components of stress. If the first requirement is discarded, and the cut is allowed to terminate inside the material, the elastic fields are singular at the edge of the cut. Dislocations of this type which are of particular interest in materials science and have recently gained applications in geophysics could justly be called singular Volterra dislocations. A simplifying feature of singular Volterra dislocations is that the strain and stress fields depend only on the boundary of the cut and 
not on its over-all shape. In addition, more complicated configurations of singular Volterra dislocations can often be obtained by superposition of simpler cases.

The idea of angular dislocations was first put forth by Yoffe [1] who showed that they can be superposed to yield any closed polygonal loop. Furthermore, she gave the elastic fields for angular dislocations in the unbounded material. However, in geophysical applications where polygonal loops are used to model faults in the crust of the earth [2], the effect of the free surface cannot be neglected and it is necessary to know the fields in a half space. The present paper presents for this purpose the displacements induced by angular dislocations in the elastic half space, while leaving the generation of results for closed polygonal loops to a later publication in a journal devoted to geophysics.

\section{Approach}

There is no loss in generality by taking one leg of the angular dislocation as perpendicular to the surface of the half space, since any other angular dislocation can be obtained by superposition. The angular dislocation considered thus lies in a plane normal to the free surface. The dislocation is also made to coincide with one of the coordinate planes $\left(y_{3}, y_{1}\right)$, as shown in Fig. 1. It may be noted that the specific configuration treated here is particularly convenient for generating closed polygonal loops.

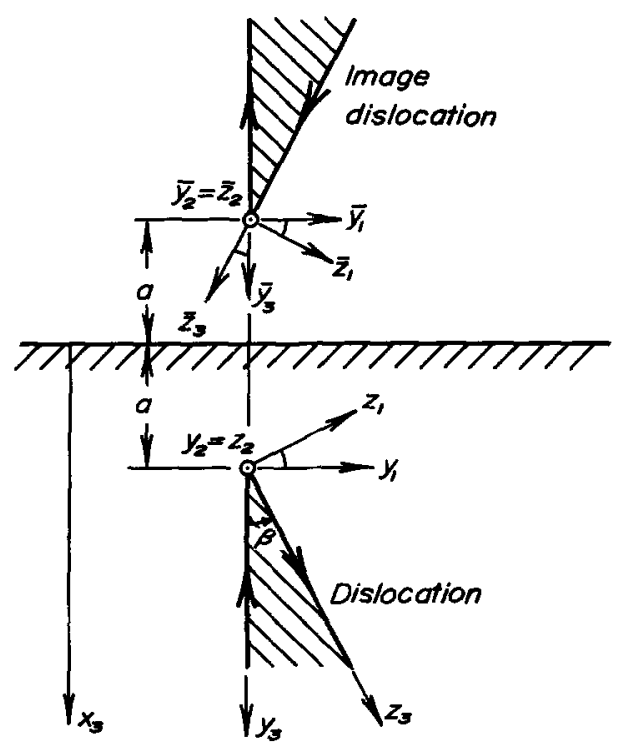

Figure 1. Angular dislocation in a half space.

The solution for any interior action in the elastic half space can be constructed by modifying the fields that would be induced by the same action in the whole space. Thus consider an angular dislocation in the infinitely extended material, placed in the region $x_{3}=y_{3}+a>0$ and having the relation to the coordinate axes indicated in Fig. 1 . The components of the Burgers vector of this dislocation in the $y_{i}$-system are $\left(B_{1}, B_{2}, B_{3}\right)$. 
The single angular dislocation in the infinitely extended material leads to tractions on the plane $x_{3}=0$ that contain both normal $\left(\tau_{33}\right)$ and shearing $\left(\tau_{23}, \tau_{31}\right)$ components. The shearing tractions on the plane $x_{3}=0$ can be eliminated, however, by putting in the infinitely extended material a second dislocation that is a mirror image of the first dislocation across the plane $x_{3}=0$ and has the direction ${ }^{1}$ indicated in Fig. 1. For the Burgers vectors $\left(B_{1}, 0,0\right)$ and $\left(0, B_{2}, 0\right)$ of the first dislocation or Cases I and II, respectively, the image dislocation has the same Burgers vector as the dislocation in $x_{3}>0$. For $\left(0,0, B_{3}\right)$ or Case III, on the other hand, the image dislocation must have the opposite Burgers vector. It also may be noted that adding the image dislocation simply doubles the normal traction on $x_{3}=0$ induced by the original dislocation. The calculations are facilitated if a coordinate system is attached to each leg of the dislocation and its image, as shown in Fig. 1. The obvious relations between the different coordinates are

$$
\begin{array}{ll}
z_{1}=y_{1} \cos \beta-y_{3} \sin \beta, & \bar{z}_{1}=y_{1} \cos \beta+\bar{y}_{3} \sin \beta, \\
z_{2}=y_{2}, & \bar{z}_{2}=y_{2}, \\
z_{3}=y_{1} \sin \beta+y_{3} \cos \beta, & \bar{z}_{3}=-y_{1} \sin \beta+\bar{y}_{3} \cos \beta, \\
R^{2}=y_{1}^{2}+y_{2}^{2}+y_{3}^{2}=z_{1}^{2}+y_{2}^{2}+z_{3}^{2}, & \bar{R}^{2}=y_{1}^{2}+y_{2}^{2}+\bar{y}_{3}^{2}=\bar{z}_{1}^{2}+y_{2}^{2}+\bar{z}_{3}^{2} .
\end{array}
$$

In order to obtain a free surface at $x_{3}=0$, the remaining task is to cancel the normal tractions induced by the given dislocation and its image. This is, however, a standard problem for the elastic half space [3] which can be treated by means of a single harmonic function $\bar{A}$ that is regular in $x_{3}>0$. The displacement derived from $\bar{A}$ is given by

$$
2 \mu v_{i}=-\left(\bar{y}_{3}-a\right) \partial_{3} \partial_{i} \bar{\Lambda}-(1-2 v) \partial_{i} \bar{\Lambda}+(3-4 v) \delta_{3 i} \partial_{3} \bar{\Lambda},
$$

where $\mu$ is the shear modulus, $v$ Poisson's ratio and the derivatives are taken with respect to the coordinates $y_{i}$ or $\bar{y}_{i}$. The tractions on $x_{3}=\bar{y}_{3}-a=0$ associated with the potential $\bar{\Lambda}$ are

$$
\tau_{23}=\tau_{31}=0, \quad \tau_{33}=\partial_{3} \partial_{3} \bar{\Lambda} .
$$

Several combinations of elementary functions appear frequently in the expressions for the field quantities, which makes it convenient to adopt the following abbreviations:

$$
\begin{aligned}
& F=-\tan ^{-1} \frac{y_{2}}{y_{1}}+\tan ^{-1} \frac{y_{2}}{z_{1}}+\tan ^{-1} \frac{y_{2} R \sin \beta}{y_{1} z_{1}+y_{2}^{2} \cos \beta}, \\
& \bar{F}=-\tan ^{-1} \frac{y_{2}}{y_{1}}+\tan ^{-1} \frac{y_{2}}{\bar{z}_{1}}+\tan ^{-1} \frac{y_{2} \bar{R} \sin \beta}{y_{1} \bar{z}_{1}+y_{2}^{2} \cos \beta}, \\
& \bar{\varphi}=-y_{2} \bar{F}-y_{1} \log \left(\bar{R}+\bar{y}_{3}\right)+\bar{z}_{1} \log \left(\bar{R}+\bar{z}_{3}\right), \\
& \bar{\chi}=-y_{1} \bar{F}+y_{2} \log \left(\bar{R}+\bar{y}_{3}\right)-y_{2} \cos \beta \log \left(\bar{R}+\bar{z}_{3}\right), \\
& \bar{\psi}=\bar{y}_{3} \bar{F}+y_{2} \sin \beta \log \left(\bar{R}+\bar{z}_{3}\right) .
\end{aligned}
$$

1 The directions assigned to the two dislocations shown in Fig. 1 are such that the discontinuity in displacement or Burgers vector is evaluated for both of them upon crossing the cut or slip plane in the direction of increasing $y_{2}$, with the understanding that the coordinate system $y_{\mathrm{i}}$ is right handed. The slip plane is the sector with the angle $\beta$. 
Finally it may be noted that the subsequent expressions for the elastic fields are restricted to the angles $-\pi / 2 \leqq \beta \leqq \pi / 2$. If $\beta$ is outside this interval, the slanting leg of the image dislocation cuts into the half space, and an additional dislocation appears in the material. It is a simple matter, however, to overcome this difficulty, as will be explained later.

\section{Case I: Burgers vector $\left(B_{1}, 0,0\right)$}

The displacement induced in the infinitely extended material by the given dislocation and its image is obtained by simply superposing the fields given by Yoffe [1]. The components of this displacement in the coordinate system $y_{i}$ are

$$
\begin{aligned}
& \frac{8 \pi(1-v)}{B_{1}} v_{1}^{\infty}=2(1-v)(F+\bar{F})-y_{1} y_{2}\left\{\frac{1}{R\left(R-y_{3}\right)}+\frac{1}{\bar{R}\left(\bar{R}+\bar{y}_{3}\right)}\right\} \\
&-y_{2} \cos \beta\left\{\frac{R \sin \beta-y_{1}}{R\left(R-z_{3}\right)}+\frac{\bar{R} \sin \beta-y_{1}}{\bar{R}\left(\bar{R}+\bar{z}_{3}\right)}\right\},
\end{aligned}
$$

$$
\begin{aligned}
\frac{8 \pi(1-v)}{B_{1}} v_{2}^{\infty}= & (1-2 v)\left\{\log \left(R-y_{3}\right)+\log \left(\bar{R}+\bar{y}_{3}\right)-\cos \beta\left[\log \left(R-z_{3}\right)+\log \left(\bar{R}+\bar{z}_{3}\right)\right]\right\} \\
& -y_{2}^{2}\left\{\frac{1}{R\left(R-y_{3}\right)}+\frac{1}{\bar{R}\left(\bar{R}+\bar{y}_{3}\right)}-\cos \beta\left[\frac{1}{R\left(R-z_{3}\right)}+\frac{1}{\bar{R}\left(\bar{R}+\bar{z}_{3}\right)}\right]\right\} \\
\frac{8 \pi(1-v)}{B_{1}} v_{3}^{\infty}= & y_{2}\left\{\frac{1}{R}-\frac{1}{\bar{R}}-\cos \beta\left[\frac{R \cos \beta-y_{3}}{R\left(R-z_{3}\right)}-\frac{\bar{R} \cos \beta+\bar{y}_{3}}{\bar{R}\left(\bar{R}+\bar{z}_{3}\right)}\right]\right\}
\end{aligned}
$$

The normal traction on the plane $x_{3}=y_{3}+a=0$ due to the dislocation and its image is ${ }^{2}$

$$
\begin{aligned}
\frac{2 \pi(1-v)}{\mu B_{1}}\left(\tau_{33}^{\infty}\right)_{x_{3}=0}=\frac{y_{2}}{R}\left\{-\left(\frac{1}{R+a}-\frac{\cos \beta}{R-z_{3}}\right)\left(1-2 v-\frac{a^{2}}{R^{2}}\right)\right. & \\
+ & \left.\frac{1}{R}\left[1-\frac{(R \cos \beta+a)^{2} \cos \beta}{\left(R-z_{3}\right)^{2}}\right]\right\} .
\end{aligned}
$$

The potential that is related to the displacement through (2) and clears the surface $x_{3}=y_{3}+a=0$ of the tractions given by (8) is

$$
\begin{aligned}
\frac{2 \pi(1-v)}{\mu B_{1}} \bar{\Lambda}=y_{2}\left\{v \log \left(\bar{R}+\bar{y}_{3}\right)\right. & +\frac{v \bar{y}_{3}-a}{\left.\bar{R}+\bar{y}_{3}\right\}} \\
& -\cot \beta\left\{a \frac{\partial \bar{\varphi}}{\partial y_{2}}+2(1-v) \bar{\chi} \cot \beta-(1-2 v) \bar{\psi}\right\} .
\end{aligned}
$$

The components of displacement derived from the given potential $\bar{A}$ and which constitute the corrective part of the solution are in the $y_{i}$ coordinate system

2 The derivations for angular dislocations and related configurations are greatly facilitated through the tables provided by Dixon [4]. 


$$
\begin{gathered}
\frac{4 \pi(1-v)}{B_{1}} v_{1}^{c}=-2(1-v)(1-2 v) \bar{F} \cot ^{2} \beta+\frac{(1-2 v) y_{2}}{\bar{R}+\bar{y}_{3}}\left\{\left(1-2 v-\frac{a}{\bar{R}}\right) \cot \beta\right. \\
\left.-\frac{y_{1}}{\bar{R}+\bar{y}_{3}}\left(v+\frac{a}{\bar{R}}\right)\right\}+\frac{(1-2 v) y_{2} \cos \beta \cot \beta}{\bar{R}+\bar{z}_{3}}\left(\cos \beta+\frac{a}{\bar{R}}\right)+\frac{a y_{2}\left(\bar{y}_{3}-a\right) \cot \beta}{\bar{R}^{3}} \\
+\frac{y_{2}\left(\bar{y}_{3}-a\right)}{\bar{R}\left(\bar{R}+\bar{y}_{3}\right)}\left\{-(1-2 v) \cot \beta+\frac{y_{1}}{\bar{R}+\bar{y}_{3}}\left(2 v+\frac{a}{\bar{R}}\right)+\frac{a y_{1}}{\bar{R}^{2}}\right\} \\
+\frac{y_{2}\left(\bar{y}_{3}-a\right)}{\bar{R}\left(\bar{R}+\bar{z}_{3}\right)}\left\{\begin{array} { l } 
{ \operatorname { c o s } \beta } \\
{ \overline { R } + \overline { z } _ { 3 } }
\end{array} \left[\left(\bar{R} \cos \beta+\bar{y}_{3}\right)\left((1-2 v) \cos \beta-\frac{a}{\bar{R}}\right) \cot \beta\right.\right. \\
\left.\left.+2(1-v)\left(\bar{R} \sin \beta-y_{1}\right) \cos \beta\right]-\frac{a \bar{y}_{3} \cos \beta \cot \beta}{\bar{R}^{2}}\right\}
\end{gathered}
$$

$$
\frac{4 \pi(1-v)}{B_{1}} v_{2}^{c}=(1-2 v)\left\{\left[2(1-v) \cot ^{2} \beta-v\right] \log \left(\bar{R}+\bar{y}_{3}\right)\right.
$$$$
\left.-\left[2(1-v) \cot ^{2} \beta+1-2 v\right] \cos \beta \log \left(\bar{R}+\bar{z}_{3}\right)\right\}
$$$$
-\frac{1-2 v}{\bar{R}+\bar{y}_{3}}\left\{y_{1} \cot \beta\left(1-2 v-\frac{a}{\bar{R}}\right)+v \bar{y}_{3}-a+\frac{y_{2}^{2}}{\bar{R}+\bar{y}_{3}}\left(v+\frac{a}{\bar{R}}\right)\right\}
$$$$
-\frac{(1-2 v) \bar{z}_{1} \cot \beta}{\bar{R}+\bar{z}_{3}}\left(\cos \beta+\frac{a}{\bar{R}}\right)-\frac{a y_{1}\left(\bar{y}_{3}-a\right) \cot \beta}{\bar{R}^{3}}
$$$$
+\frac{\bar{y}_{3}-a}{\bar{R}+\bar{y}_{3}}\left\{-2 v+\frac{1}{\bar{R}}\left[(1-2 v) y_{1} \cot \beta-a\right]+\frac{y_{2}^{2}}{\bar{R}\left(\bar{R}+\bar{y}_{3}\right)}\left(2 v+\frac{a}{\bar{R}}\right)+\frac{a y_{2}^{2}}{\bar{R}^{3}}\right\}
$$$$
+\frac{\bar{y}_{3}-a}{\bar{R}+\bar{z}_{3}}\left\{\cos ^{2} \beta-\frac{1}{\bar{R}}\left[(1-2 v) \bar{z}_{1} \cot \beta+a \cos \beta\right]+\begin{array}{c}
a \bar{y}_{3} \bar{z}_{1} \cot \beta \\
\bar{R}^{3}
\end{array}\right.
$$$$
\left.-\frac{1}{\bar{R}\left(\bar{R}+\bar{z}_{3}\right)}\left[y_{2}^{2} \cos ^{2} \beta-\frac{a \bar{z}_{1} \cot \beta}{\bar{R}}\left(\bar{R} \cos \beta+\bar{y}_{3}\right)\right]\right\}
$$

$$
\begin{aligned}
& \frac{4 \pi(1-v)}{B_{1}} v_{3}^{c}=2(1-v)\left\{(1-2 v) \bar{F} \cot \beta+\frac{y_{2}}{\bar{R}+\bar{y}_{3}}\left(2 v+\frac{a}{\bar{R}}\right)\right. \\
& \left.\quad-\frac{y_{2} \cos \beta}{\bar{R}+\bar{z}_{3}}\left(\cos \beta+\frac{a}{\bar{R}}\right)\right\}+\frac{y_{2}\left(\bar{y}_{3}-a\right)}{\bar{R}}\left(\frac{2 v}{\bar{R}+\bar{y}_{3}}+\frac{a}{\bar{R}^{2}}\right) \\
& \quad+\frac{y_{2}\left(\bar{y}_{3}-a\right) \cos \beta}{\bar{R}\left(\bar{R}+\bar{z}_{3}\right)}\left\{1-2 v-\frac{\bar{R} \cos \beta+\bar{y}_{3}}{\bar{R}+\bar{z}_{3}}\left(\cos \beta+\frac{a}{\bar{R}}\right)-\frac{a \bar{y}_{3}}{\bar{R}^{2}}\right\} .
\end{aligned}
$$

The total displacement is evidently

$v_{i}=v_{i}^{\infty}+v_{i}^{c}$. 


\section{Case II: Burgers vector $\left(0, B_{2}, 0\right)$}

Using the same notation as before, the results for this case are

$$
\begin{aligned}
& \frac{8 \pi(1-v)}{B_{2}} v_{1}^{\infty}=-(1-2 v)\left\{\log \left(R-y_{3}\right)+\log \left(\bar{R}+\bar{y}_{3}\right)-\cos \beta\left[\log \left(R-z_{3}\right)+\log \left(\bar{R}+\bar{z}_{3}\right)\right]\right\} \\
& +y_{1}^{2}\left\{\frac{1}{R\left(R-y_{3}\right)}+\frac{1}{\bar{R}\left(\overline{\bar{R}}+\bar{y}_{3}\right)}\right\}+\frac{z_{1}\left(R \sin \beta-y_{1}\right)}{R\left(R-z_{3}\right)}+\frac{\bar{z}_{1}\left(\bar{R} \sin \beta-y_{1}\right)}{\bar{R}\left(\bar{R}+\bar{z}_{3}\right)}, \\
& \frac{8 \pi(1-v)}{B_{2}} v_{2}^{\infty}=2(1-v)(F+\bar{F})+y_{1} y_{2}\left\{\frac{1}{R\left(R-y_{3}\right)}+\frac{1}{\bar{R}\left(\bar{R}+\bar{y}_{3}\right)}\right\} \\
& -y_{2}\left\{\frac{z_{1}}{R\left(R-z_{3}\right)}+\frac{\bar{z}_{1}}{\bar{R}\left(\bar{R}+\bar{z}_{3}\right)}\right\},
\end{aligned}
$$

$\frac{8 \pi(1-v)}{B_{2}} v_{3}^{\infty}=-(1-2 v) \sin \beta\left\{\log \left(R-z_{3}\right)-\log \left(\bar{R}+\bar{z}_{3}\right)\right\}$

$$
-y_{1}\left(\frac{1}{R}-\frac{1}{\bar{R}}\right)+\frac{z_{1}\left(R \cos \beta-y_{3}\right)}{R\left(R-z_{3}\right)}-\frac{\bar{z}_{1}\left(\bar{R} \cos \beta+\bar{y}_{3}\right)}{\bar{R}\left(\bar{R}+\bar{z}_{3}\right)},
$$

$$
\begin{aligned}
\frac{2 \pi(1-v)}{\mu B_{2}}\left(\tau_{33}^{\infty}\right)_{x_{3}=0}=-\frac{y_{1}}{R}\left(\frac{2 v}{R+a}+\frac{a}{R^{2}}\right) & -\frac{1}{R\left(R-z_{3}\right)}\left\{2 \cos \beta\left(R \sin \beta-y_{1}\right)\right. \\
+ & \left.\frac{z_{1}}{R}\left[\frac{R^{2} \cdot-a^{2}}{R}-\frac{(R \cos \beta+a)^{2}}{R-z_{3}}\right]\right\},
\end{aligned}
$$

$$
\begin{aligned}
& \frac{2 \pi(1-v)}{\mu B_{2}} \bar{\Lambda}=-y_{1}\left\{v \log \left(\bar{R}+\bar{y}_{3}\right)+\frac{v \bar{y}_{3}-a}{\bar{R}+\bar{y}_{3}}\right\}+a \frac{\partial \bar{\varphi}}{\partial y_{3}} \\
& \quad+\cot \beta\left\{(1-2 v)\left[\bar{R}-\bar{y}_{3} \log \left(\bar{R}+\bar{y}_{3}\right)\right]+\bar{R}-\bar{z}_{3} \log \left(\bar{R}+\bar{z}_{3}\right)\right\}+a \frac{\partial \bar{\varphi}}{\partial y_{1}}+2(1-v) \bar{\varphi} \cot \beta
\end{aligned}
$$$$
\frac{4 \pi(1-v)}{B_{2}} v_{1}^{c}=(1-2 v)\left\{\left[2(1-v) \cot ^{2} \beta+v\right] \log \left(\bar{R}+\bar{y}_{3}\right)\right.
$$$$
\left.-\left[2(1-v) \cot ^{2} \beta+1\right] \cos \beta \log \left(\bar{R}+\bar{z}_{3}\right)\right\}
$$$$
+\frac{1-2 v}{\bar{R}+\bar{y}_{3}}\left\{-(1-2 v) y_{1} \cot \beta+v \bar{y}_{3}-a+\frac{a y_{1} \cot \beta}{\bar{R}}+\frac{y_{1}^{2}}{\bar{R}+\bar{y}_{3}}\left(v+\frac{a}{\bar{R}}\right)\right\}
$$$$
-\frac{(1-2 v) \cot \beta}{\bar{R}+\bar{z}_{3}}\left\{\bar{z}_{1} \cos \beta-\frac{a\left(\bar{R} \sin \beta-y_{1}\right)}{\bar{R} \cos \beta}\right\}-\frac{a y_{1}\left(\bar{y}_{3}-a\right) \cot \beta}{\bar{R}^{3}}
$$$$
+\frac{\bar{y}_{3}-a}{\bar{R}+\bar{y}_{3}}\left\{2 v+\frac{1}{\bar{R}}\left[(1-2 v) y_{1} \cot \beta+a\right]-\frac{y_{1}^{2}}{\bar{R}\left(\bar{R}+\bar{y}_{3}\right)}\left(2 v+\frac{a}{\bar{R}}\right)-\frac{a y_{1}^{2}}{\bar{R}^{3}}\right\}
$$ 


$$
\begin{aligned}
& +\frac{\left(\bar{y}_{3}-a\right) \cot \beta}{\bar{R}+\bar{z}_{3}}\left\{-\cos \beta \sin \beta+\frac{a y_{1} \bar{y}_{3}}{\bar{R}^{3} \cos \beta}\right. \\
& \left.+\frac{\bar{R} \sin \beta-y_{1}}{\bar{R}}\left[2(1-v) \cos \beta-\frac{\bar{R} \cos \beta+\bar{y}_{3}}{\bar{R}+\bar{z}_{3}}\left(1+\frac{a}{\bar{R} \cos \beta}\right)\right]\right\}, \\
& \frac{4 \pi(1-v)}{B_{2}} v_{2}^{c}=2(1-v)(1-2 v) \bar{F} \cot ^{2} \beta+\frac{(1-2 v) y_{2}}{\tilde{R}+\bar{y}_{3}}\left\{-\left(1-2 v-\frac{a}{\bar{R}}\right) \cot \beta\right. \\
& \left.+\frac{y_{1}}{\bar{R}+\bar{y}_{3}}\left(v+\frac{a}{\bar{R}}\right)\right\}-\frac{(1-2 v) y_{2} \cot \beta}{\bar{R}+\bar{z}_{3}}\left(1+\frac{a}{\bar{R} \cos \beta}\right)-\frac{a y_{2}\left(\bar{y}_{3}-a\right) \cot \beta}{\bar{R}^{3}} \\
& \left.+\frac{y_{2}\left(\bar{y}_{3}-a\right)}{\bar{R}(\bar{R}}+\bar{y}_{3}\right)\left\{(1-2 v) \cot \beta-\frac{2 v y_{1}}{\bar{R}+\bar{y}_{3}}-\frac{a y_{1}}{\bar{R}}\left(\frac{1}{\bar{R}}+\frac{1}{\bar{R}+\bar{y}_{3}}\right)\right\} \\
& +\frac{y^{2}\left(\bar{y}_{3}-a\right) \cot \beta}{\bar{R}\left(\bar{R}+\bar{z}_{3}\right)}\left\{-2(1-v) \cos \beta+\frac{\bar{R} \cos \beta+\bar{y}_{3}}{\bar{R}+\bar{z}_{3}}\left(1+\frac{a}{\bar{R} \cos \beta}\right)+\frac{a \bar{y}_{3}}{\bar{R}^{2} \cos \beta}\right\}, \\
& \frac{4 \pi(1-v)}{B_{2}} v_{3}^{c}=-2(1-v)(1-2 v) \cot \beta\left\{\log \left(\bar{R}+\bar{y}_{3}\right)-\cos \beta \log \left(\bar{R}+\bar{z}_{3}\right)\right\} \\
& -\frac{2(1-v) y_{1}}{\bar{R}+\bar{y}_{3}}\left(2 v+\frac{a}{\bar{R}}\right)+\frac{2(1-v) \bar{z}_{1}}{\bar{R}+\bar{z}_{3}}\left(\cos \beta+\frac{a}{\bar{R}}\right) \\
& +\frac{\bar{y}_{3}-a}{\bar{R}}\left\{(1-2 v) \cot \beta-\frac{2 v y_{1}}{\bar{R}+\bar{y}_{3}}-\frac{a y_{1}}{\bar{R}^{2}}\right\} \\
& -\frac{\bar{y}_{3}-a}{\bar{R}+\bar{z}_{3}}\left\{\cos \beta \sin \beta+\frac{\left(\bar{R} \cos \beta+\bar{y}_{3}\right) \cot \beta}{\bar{R}}\left[2(1-v) \cos \beta-\frac{\bar{R} \cos \beta+\bar{y}_{3}}{\bar{R}+\bar{z}_{3}}\right]\right. \\
& \left.+\frac{a}{\bar{R}}\left[\sin \beta-\frac{\bar{y}_{3} \bar{z}_{1}}{\bar{R}^{2}}-\frac{\bar{z}_{1}\left(\bar{R} \cos \beta+\bar{y}_{3}\right)}{\bar{R}\left(\bar{R}+\bar{z}_{3}\right)}\right]\right\} \text {. }
\end{aligned}
$$

\section{Case III : Burgers vector $\left(0,0, B_{3}\right)$}

The solution for this case is specified by the following expressions:

$$
\begin{aligned}
& \frac{8 \pi(1-v)}{B_{3}} v_{1}^{\infty}=y_{2} \sin \beta\left\{\frac{R \sin \beta-y_{1}}{R\left(R-z_{3}\right)}+\frac{\bar{R} \sin \beta-y_{1}}{\bar{R}\left(\bar{R}+\bar{z}_{3}\right)}\right\}, \\
& \frac{8 \pi(1-v)}{B_{3}} v_{2}^{\infty}=(1-2 v) \sin \beta\left\{\log \left(R-z_{3}\right)+\log \left(R+\bar{z}_{3}\right)\right\} \\
& -y_{2}^{2} \sin \beta\left\{\frac{1}{R\left(R-z_{3}\right)}+\frac{1}{\bar{R}\left(\bar{R}-\bar{z}_{3}\right)}\right\} \text {, } \\
& 8 \pi(1-v) v_{3}^{\infty}=2(1-v)(F-\bar{F})+y_{2} \sin \beta\left\{\frac{R \cos \beta-y_{3}}{R\left(R-z_{3}\right)}-\frac{\bar{R} \cos \beta+\bar{y}_{3}}{\bar{R}\left(\bar{R}+\bar{z}_{3}\right)}\right\},
\end{aligned}
$$




$$
\begin{aligned}
\frac{2 \pi(1-v)}{\mu B_{3}}\left(\tau_{33}^{\infty}\right)_{x_{3}=0}= & \frac{y_{2} \sin \beta}{R^{2}\left(R-z_{3}\right)}\left\{\frac{R^{2}+a^{2}}{R}+\frac{(R \cos \beta+a)^{2}}{R-z_{3}}\right\}, \\
\frac{2 \pi(1-v)}{\mu B_{3}} \bar{\Lambda}= & a \frac{\partial \bar{\varphi}}{\partial y_{2}}+\bar{\psi} \\
\frac{4 \pi(1-v)}{B_{3}} v_{1}^{c}= & (1-2 v)\left\{\frac{y_{2}}{\bar{R}+\bar{y}_{2}}\left(1+\frac{a}{\bar{R}}\right)-\frac{y^{2} \cos \beta}{\bar{R}+\bar{z}_{3}}\left(\cos \beta+\frac{a}{\bar{R}}\right)\right\} \\
& -\frac{y_{2}\left(\bar{y}_{3}-a\right)}{\bar{R}}\left(\frac{a}{\bar{R}^{2}}+\frac{1}{\bar{R}+\bar{y}_{3}}\right) \\
& +\frac{y_{2}\left(\bar{y}_{3}-a\right) \cos \beta}{\bar{R}\left(\bar{R}+\bar{z}_{3}\right)}\left\{\frac{\bar{R} \cos \beta+\bar{y}_{3}}{\bar{R}+\bar{z}_{3}}\left(\cos \beta+\frac{a}{\bar{R}}\right)+\frac{a \bar{y}_{3}}{\bar{R}^{2}}\right\},
\end{aligned}
$$

$$
\begin{aligned}
\frac{4 \pi(1-v)}{B_{3}} v_{2}^{c}= & (1-2 v)\left\{-\sin \beta \log \left(\bar{R}+\bar{z}_{3}\right)-\frac{y_{1}}{\bar{R}+\bar{y}_{3}}\left(1+\frac{a}{\bar{R}}\right)+\frac{\bar{z}_{1}}{\bar{R}+\bar{y}_{3}}\left(\cos \beta+\frac{a}{\bar{R}}\right)\right\} \\
& +\frac{y_{1}\left(\bar{y}_{3}-a\right)}{\bar{R}}\left(\frac{a}{\bar{R}^{2}}+\frac{1}{\bar{R}+\bar{y}_{3}}\right) \\
& -\frac{\bar{y}_{3}-a}{\bar{R}+\bar{z}_{3}}\left\{\sin \beta\left(\cos \beta-\frac{a}{\bar{R}}\right)+\frac{\bar{z}_{1}}{\bar{R}}\left(1+\frac{a \bar{y}_{3}}{\bar{R}^{2}}\right)\right. \\
& \left.-\frac{1}{\bar{R}\left(\bar{R}+\bar{z}_{3}\right)}\left[y_{2}^{2} \cos \beta \sin \beta-\frac{a \bar{z}_{1}}{\bar{R}}\left(\bar{R} \cos \beta+\bar{y}_{3}\right)\right]\right\}
\end{aligned}
$$

$$
\begin{aligned}
\frac{4 \pi(1-v)}{B_{3}} v_{3}^{c}= & 2(1-v)\left\{F+\frac{y_{2} \sin \beta}{\bar{R}+\bar{z}_{3}}\left(\cos \beta+\frac{a}{\bar{R}}\right)\right\} \\
& +\frac{y_{2}\left(\bar{y}_{3}-a\right) \sin \beta}{\bar{R}\left(\bar{R}+\bar{z}_{3}\right)}\left\{1+\frac{\bar{R} \cos \beta+\bar{y}_{3}}{\bar{R}+\bar{z}_{3}}\left(\cos \beta+\frac{a}{\bar{R}}\right)+\frac{a \bar{y}_{3}}{\bar{R}^{2}}\right\} .
\end{aligned}
$$

\section{Displacement at the surface}

Of particular interest in geophysical applications is the displacement at the free surface, because it is the most readily measured quantity. It can be shown that the displacement at the surface depends linearly on Poisson's ratio for any dislocation configuration [5]. ${ }^{3}$ It requires, however, some fairly tedious manipulations to bring out this dependence starting with the general expressions given in the previous sections, and it is worth while to record the results.

\footnotetext{
${ }^{3}$ The special dependence of the surface data on Poisson's ratio was first observed by Converse for rectangular loops [6].
} 
Case I:

$$
\begin{aligned}
\frac{2 \pi}{B_{1}} v_{1}= & \left\{1-(1-2 v) \cot ^{2} \beta\right\} F+\frac{y_{2}}{R+a}\left\{(1-2 v)\left[\cot \beta+\frac{y_{1}}{2(R+a)}\right]-\frac{y_{1}}{R}\right\} \\
& -\frac{y^{2}\left(R \sin \beta-y_{1}\right) \cos \beta}{R\left(R-z_{3}\right)},
\end{aligned}
$$

$$
\begin{aligned}
\frac{2 \pi}{B_{1}} v_{2}= & (1-2 v)\left\{\left(\frac{1}{2}+\cot ^{2} \beta\right) \log (R+a)-\frac{\cot \beta}{\sin \beta} \log \left(R-z_{3}\right)\right\} \\
& -\frac{1}{R+a}\left\{(1-2 v)\left[y_{1} \cot \beta-\frac{1}{2} a-\frac{y_{2}^{2}}{2(R+a)}\right]+\frac{y_{2}^{2}}{R}\right\}+\frac{y_{2}^{2} \cos \beta}{R\left(R-z_{3}\right)},
\end{aligned}
$$

$\frac{2 \pi}{B_{1}} v_{3}=(1-2 v) F \cot \beta+\frac{y_{2}}{R+a}\left(2 v+\frac{a}{R}\right)-\frac{y_{2} \cos \beta}{R-z_{3}}\left(\cos \beta+\frac{a}{R}\right)$.

Case II:

$$
\begin{aligned}
\frac{2 \pi}{B_{2}} v_{1} & =-(1-2 v)\left\{\left(\frac{1}{2}-\cot ^{2} \beta\right) \log (R+a)+\cos \beta \cot ^{2} \beta \log \left(R-z_{3}\right)\right\} \\
& -\frac{1}{R+a}\left\{(1-2 v)\left[y_{1} \cot \beta+\frac{1}{2} a+\frac{y_{1}^{2}}{2(R+a)}\right]-\frac{y_{1}^{2}}{R}\right\}+\frac{z_{1}\left(R \sin \beta-y_{1}\right)}{R\left(R-z_{3}\right)}, \\
\frac{2 \pi}{B_{2}} v_{2}=\left\{1+(1-2 v) \cot ^{2} \beta\right\} F-\frac{y_{2}}{R+a}\left\{(1-2 v)\left[\cot \beta+\frac{y_{1}}{2(R+a)}\right]-\frac{y_{1}}{R}\right\} & -\frac{y_{2} z_{1}}{R\left(R-z_{3}\right)},
\end{aligned}
$$

$\frac{2 \pi}{B_{2}} v_{3}=-(1-2 v) \cot \beta\left\{\log (R+a)-\cos \beta \log \left(R-z_{3}\right)\right\}$

$$
-\frac{y_{1}}{R+a}\left(2 v+\frac{a}{R}\right)+\frac{z_{1}}{R-z_{3}}\left(\cos \beta+\frac{a}{R}\right) .
$$

Case III:

$$
\begin{aligned}
& \frac{2 \pi}{B_{3}} v_{1}=\frac{y_{2}\left(R \sin \beta-y_{1}\right) \sin \beta}{R\left(R-z_{3}\right)}, \\
& \frac{2 \pi}{B_{3}} v_{2}=-\frac{y_{2}^{2} \sin \beta}{R\left(R-z_{3}\right)}, \\
& \frac{2 \pi}{B_{3}} v_{3}=F+\frac{y_{2}(R \cos \beta+a) \sin \beta}{R\left(R-z_{3}\right)} .
\end{aligned}
$$

It may be noted that for Case III the displacement at the surface is independent of the 
elastic constants.

In all expressions pertaining to the free surface,

$$
\begin{aligned}
& z_{1}=y_{1} \cos \beta+a \sin \beta, \\
& z_{3}=y_{1} \sin \beta-a \cos \beta, \\
& R^{2}=y_{1}^{2}+y_{2}^{2}+a^{2} .
\end{aligned}
$$

\section{Strain at the surface}

The strain in the $y_{i}$ coordinate system is

$$
\eta_{i j}=\frac{1}{2}\left(\frac{\partial v_{j}}{\partial y_{i}}+\frac{\partial v_{i}}{\partial y_{j}}\right)
$$

The components $\eta_{23}$ and $\eta_{31}$ vanish at the free surface on account of the boundary conditions. The remaining components at the surface $\left(x_{3}=y_{3}+a=0\right)$ are given below.

Case I:

$$
\begin{aligned}
& \frac{2 \pi}{B_{1}} \eta_{11}= \frac{y_{2}}{R+a}\left\{(1-2 v)\left[-\frac{\cot ^{2} \beta}{R}+\frac{1}{2(R+a)}-\frac{y_{1}}{R(R+a)}\left(\cot \beta+\frac{y_{1}}{R+a}\right)\right]\right. \\
&\left.+\frac{y_{1}^{2}}{R^{2}}\left(\frac{1}{R}+\frac{1}{R+a}\right)\right\}+\frac{y_{2} \cos \beta}{R\left(R-z_{3}\right)}\left\{(1-2 v) \cot ^{2} \beta-\frac{y_{1}^{2}}{R^{2}}-\frac{\left(R \sin \beta-y_{1}\right)^{2}}{R\left(R-z_{3}\right)}\right\}, \\
& \frac{2 \pi}{B_{1}} \eta_{22}= \frac{y_{2}}{R+a}\left\{-\left[2-(1-2 v) \cot ^{2} \beta\right] \frac{1}{R}+\frac{y_{2}^{2}}{R^{2}}\left(\frac{1}{R}+\frac{1}{R+a}\right)\right. \\
&\left.+\frac{1-2 v}{R+a}\left[\frac{3}{2}+\frac{y_{1} \cot \beta}{R}-\frac{y_{2}^{2}}{R(R+a)}\right]\right\} \\
&+\frac{y_{2} \cos \beta}{R\left(R-z_{3}\right)}\left\{1+2 v-(1-2 v) \cot ^{2} \beta-\frac{y_{2}^{2}}{R}\left(\frac{1}{R}+\frac{1}{R-z_{3}}\right)\right\},
\end{aligned}
$$

$$
\frac{2 \pi}{B_{1}} \eta_{33}=\frac{v}{1-v}\left\{-\frac{y_{2}}{R}\left(\frac{1-2 v}{R+a}-\frac{a}{R^{2}}\right)+\frac{y_{2} \cos \beta}{R\left(R-z_{3}\right)}\left[2(1-v)-\frac{a^{2}}{R^{2}}-\frac{(R \cos \beta+a)^{2}}{R\left(R-z_{3}\right)}\right]\right\}
$$

$$
\begin{aligned}
\frac{2 \pi}{B_{1}} \eta_{12}= & \frac{1}{R+a}\left\{-\left[1-(1-2 v) \cot ^{2} \beta\right] \frac{y_{1}}{R}+\frac{y_{1} y_{2}^{2}}{R^{2}}\left(\frac{1}{R}+\frac{1}{R+a}\right)\right. \\
& \left.+\frac{1-2 v}{R+a}\left[\frac{1}{2} y_{1}+\frac{\left(y_{1}^{2}-y_{2}^{2}\right) \cot \beta}{2 R}-\frac{y_{1} y_{2}^{2}}{R(R+a)}\right]\right\}
\end{aligned}
$$


$+\frac{1}{R\left(R-z_{3}\right)}\left\{\frac{1}{2}\left[1-(1-2 v) \cot ^{2} \beta\right] z_{1}-\left[v-\frac{1}{2}(1-2 v) \cot ^{2} \beta\right]\left(R \sin \beta-y_{1}\right) \cos \beta\right.$

Case II:

$$
\left.-\frac{y_{2}^{2} \cos \beta}{R}\left(\frac{y_{1}}{R}-\frac{R \sin \beta-y_{1}}{R-z_{3}}\right)\right\} \text {. }
$$

$$
\begin{aligned}
\frac{2 \pi}{B_{2}} \eta_{11}= & \frac{1}{R+a}\left\{\left[2+(1-2 v) \cot ^{2} \beta\right] \frac{y_{1}}{R}-\frac{y_{1}^{3}}{R^{2}}\left(\frac{1}{R}+\frac{1}{R+a}\right)\right. \\
& \left.-(1-2 v)\left[\cot \beta+\frac{y_{1}}{R+a}\left(\frac{3}{2}-\frac{y_{1} \cot \beta}{R}-\frac{y_{1}^{2}}{R(R+a)}\right)\right]\right\} \\
& +\frac{1}{R\left(R-z_{3}\right)}\left\{\left[1+(1-2 v) \cot ^{2} \beta\right](R \cos \beta+a) \sin \beta-\left[2+(1-2 v) \cot ^{2} \beta\right] z_{1}\right.
\end{aligned}
$$

$$
\left.+\frac{y_{1}^{2} z_{1}}{R^{2}}+\frac{z_{1}\left(R \sin \beta-y_{1}\right)^{2}}{R\left(R-z_{3}\right)}\right\}
$$

$$
\begin{aligned}
\frac{2 \pi}{B_{2}} \eta_{22}=- & \frac{1}{R+a}\left\{( 1 - 2 v ) \left[\cot \beta+\frac{y_{1} \cot ^{2} \beta}{R}\right.\right. \\
-\frac{1}{R+a}\left(\frac{1}{2} y_{1}-\right. & \left.\left.\left.\frac{y_{2}^{2} \cot \beta}{R}-\frac{y_{1} y_{2}^{2}}{R(R+a)}\right)\right]+\frac{y_{1} y_{2}^{2}}{R^{2}}\left(\frac{1}{R}+\frac{1}{R+a}\right)\right\} \\
& +\frac{z_{1}}{R\left(R-z_{3}\right)}\left\{(1-2 v) \cot ^{2} \beta+\frac{y_{2}^{2}}{R}\left(\frac{1}{R}+\frac{1}{R-z_{3}}\right)\right\},
\end{aligned}
$$

$\frac{2 \pi}{B_{2}} \eta_{33}=\frac{v}{1-v}\left\{\frac{y_{1}}{R}\left(\frac{1-2 v}{R+a}-\frac{a}{R^{2}}\right)-\frac{1}{R\left(R-z_{3}\right)}\left[2(1-v) z_{1}+2 v(R \cos \beta+a) \sin \beta\right.\right.$

$$
\left.\left.-\frac{a^{2} z_{1}}{R^{2}}-\frac{z_{1}(R \cos \beta+a)^{2}}{R\left(R-z_{3}\right)}\right]\right\}
$$

$$
\begin{aligned}
& \frac{2 \pi}{B_{2}} \eta_{12}=\frac{y_{2}}{R+a}\left\{\left[1+(1-2 v) \cot ^{2} \beta\right] \frac{1}{R}-\frac{y_{1}^{2}}{R^{2}}\left(\frac{1}{R}+\frac{1}{R+a}\right)\right.\left.r \frac{1-2 v}{R+a}\left[-\frac{1}{2}+\frac{y_{1} \cot \beta}{R}+\frac{y_{1}^{2}}{R(R+a)}\right]\right\} \\
&-\frac{y_{2}}{R\left(R-z_{3}\right)}\left\{\left[1+(1-2 v) \cot ^{2} \beta\right] \cos \beta\right. \\
&\left.-\frac{y_{1} z_{1}}{R^{2}}+\frac{z_{1}\left(R \sin \beta-y_{1}\right)}{R\left(R-z_{3}\right)}\right\}
\end{aligned}
$$

Case III:

$$
\begin{aligned}
& \frac{2 \pi}{B_{3}} \eta_{11}=-\frac{y^{2} \sin \beta}{R\left(R-z_{3}\right)}\left\{1-\frac{y_{1}^{2}}{R^{2}}-\frac{\left(R \sin \beta-y_{1}\right)^{2}}{R\left(R-z_{3}\right)}\right\}, \\
& \frac{2 \pi}{B_{3}} \eta_{22}=-\frac{y_{2} \sin \beta}{R\left(R-z_{3}\right)}\left\{2-\frac{y_{2}^{2}}{R}\left(\frac{1}{R}+\frac{1}{R-z_{3}}\right)\right\},
\end{aligned}
$$




$$
\begin{aligned}
& \frac{2 \pi}{B_{3}} \eta_{33}=\frac{v y_{2} \sin \beta}{(1-v) R^{2}\left(R-z_{3}\right)}\left\{\frac{a^{2}}{R}+\frac{(R \cos \beta+a)^{2}}{R-z_{3}}\right\}, \\
& \frac{2 \pi}{B_{3}} \eta_{12}=\frac{\sin \beta}{R\left(R-z_{3}\right)}\left\{\left(R \sin \beta-y_{1}\right)\left[\frac{1}{2}-\frac{y_{2}^{2}}{R\left(R-z_{3}\right)}\right]+\frac{y_{1} y_{2}^{2}}{R^{2}}\right\} .
\end{aligned}
$$

If the surface of an elastic half space is subjected to normal tractions only, the normal components of strain at the surface are related through the Lamé condition. This condition gives for the corrective part of the solution

$$
\eta_{11}^{c}+\eta_{22}^{c}=\eta_{33}^{c},
$$

where $\eta_{i j}^{c}$ denotes strain derived from the displacement $v_{i}^{c}$. Since the surface of the half space is free of tractions, Hooke's law for the total strain leads to

$$
(1-2 v) \eta_{33}+v \eta_{k k}=0 \text {. }
$$

Combining (53) and (54) results in

$$
\begin{aligned}
& \eta_{11}+\eta_{22}=(1-v)\left(\eta_{k k}^{\infty}-2 \eta_{33}^{\infty}\right) \\
& \eta_{33}=v\left(2 \eta_{33}^{\infty}-\eta_{k k}^{\infty}\right)
\end{aligned}
$$

where $\eta_{i j}^{\infty}$ is strain derived from the displacement $v_{1}^{\infty}$. It is seen from (55) and (56) that part of the strain at the surface can be found without knowledge of the corrective part of the solution which is specified by the potential $\bar{A}$.

It also may be noted that the strain components $\eta_{11}, \eta_{22}$ and $\eta_{12}$ at the surface depend linearly on Poisson's ratio for Cases I and II, and contain no elastic constants for Case III.

\section{Rotation at the surface}

The rotation tensor, defined as

$$
\Omega_{i j}=\frac{1}{2}\left(\frac{\partial v_{j}}{\partial x_{i}}-\frac{\partial v_{i}}{\partial x_{j}}\right),
$$

is the antisymmetric part of the displacement gradient $\partial v_{j} / \partial x_{i}$. Although the quantities of principal interest in geophysical applications are the tilts or components with mixed subscripts of the displacement gradient at the surface, it is convenient to list the components of the rotation tensor, because they can be combined with the components of strain to yield the tilts, and fewer and shorter expressions need be given.

Case I:

$$
\begin{aligned}
\frac{4 \pi}{B_{1}} \Omega_{12}= & \frac{1}{R+a}\left\{(1-2 v) \cot \beta\left[-2+\frac{y_{1}^{2}+y_{2}^{2}}{R(R+a}\right]+\frac{2 y_{1}}{R}\right\} \\
& +\frac{1}{R\left(R-z_{3}\right)}\left\{-\left[1-(1-2 v) \cot ^{2} \beta\right] z_{1}+\left[2(1-v)+(1-2 v) \cot ^{2} \beta\right]\right. \\
& \left.\times\left(R \sin \beta-y_{1}\right) \cos \beta\right\},
\end{aligned}
$$




$$
\begin{aligned}
& \frac{2 \pi}{B_{1}} \Omega_{23}=\frac{1}{R+a}\left\{2 v-(1-2 v) \frac{y_{1} \cot \beta}{R}+\frac{a}{R}\left(1-\frac{y_{2}^{2}}{R^{2}}\right)-\frac{y_{2}^{2}}{R(R+a)}\left(2 v+\frac{a}{R}\right)\right\} \\
& +\frac{1}{R\left(R-z_{3}\right)}\left\{(1-2 v) z_{1} \cot \beta-(R \cos \beta+a) \cos \beta\right. \\
& \left.+\frac{y_{2}^{2} \cos \beta}{R-z_{3}}\left(\cos \beta+\frac{a}{R}\right)+\frac{a y_{2}^{2} \cos \beta}{R^{2}}\right\} \\
& \frac{2 \pi}{\bar{B}_{1}} \Omega_{31}=\frac{y_{2}}{R(R+a)}\left\{-(1-2 v) \cot \beta+\frac{y_{1}}{R+a}\left(2 v+\frac{a}{R}\right)+\frac{a y_{1}}{R^{2}}\right\} \\
& +\frac{y_{2} \cos \beta}{R\left(R-z_{3}\right)}\left\{(1-2 v) \cot \beta+\frac{R \sin \beta-y_{1}}{R-z_{3}}\left(\cos \beta+\frac{a}{R}\right)-\frac{a y_{1}}{R^{2}}\right\} \text {. }
\end{aligned}
$$

\section{Case II:}

$$
\begin{aligned}
& \begin{aligned}
\frac{2 \pi}{B_{2}} \Omega_{12}= & \frac{y_{2}}{R}\left(\frac{1}{R+a}-\frac{\cos \beta}{R-z_{3}}\right), \\
\frac{2 \pi}{B_{2}} \Omega_{23}= & \frac{y_{2}}{R(R+a}\left\{-(1-2 v) \cot \beta+\frac{y_{1}}{R+a}\left(2 v+\frac{a}{R}\right)+\frac{a y_{1}}{R^{2}}\right\} \\
& \quad+\frac{y_{2}}{R\left(R-z_{3}\right)}\left\{(1-2 v) \cos \beta \cot \beta-\frac{z_{1}}{R}\left(\frac{a}{R}+\frac{R \cos \beta+a}{R-z_{3}}\right)\right\}, \\
\frac{2 \pi}{B_{2}} \Omega_{31}= & \frac{1}{R+a}\left\{(1-2 v) \frac{y_{1} \cot \beta}{R}+\left[1-\frac{y_{1}^{2}}{R(R+a)}\right]\left(2 v+\frac{a}{R}\right)-\frac{a y_{1}^{2}}{R^{3}}\right\} \\
+\frac{1}{R\left(R-z_{3}\right)}\left\{\left[(1-2 v)\left(R \sin \beta-y_{1}\right) \cot \beta-(R \cos \beta+a)\right] \cos \beta\right. & \left.+z_{1}\left[\frac{a y_{1}}{R^{2}}-\frac{R \sin \beta-y_{1}}{R-z_{3}}\left(\cos \beta+\frac{a}{R}\right)\right]\right\} .
\end{aligned}
\end{aligned}
$$

Case III:

$$
\begin{aligned}
& \frac{4 \pi}{B_{3}} \Omega_{12}=-\frac{\left(R \sin \beta-y_{1}\right) \sin \beta}{R\left(R-z_{3}\right)} \\
& \frac{2 \pi}{B_{3}} \Omega_{23}=-\frac{y_{1}}{R^{2}}\left\{1+\frac{a^{2}}{R(R+a)}\right\}+\frac{z_{1}}{R^{2}\left(R-z_{3}\right)}\left\{\frac{a^{2}}{R}+\frac{(R \cos \beta+a)^{2}}{R-z_{3}}\right\} \\
& \frac{2 \pi}{B_{3}} \Omega_{31}=-\frac{y_{2}}{R}\left\{\frac{1}{R+a}+\frac{a}{R^{2}}-\frac{\cos \beta}{R\left(R-z_{3}\right)}\left[\frac{(R \cos \beta+a)^{2}}{R-z_{3}}+\frac{a^{2}}{R}\right]\right\}
\end{aligned}
$$




\section{Conclusion}

As mentioned before, the formulas given for the elastic fields of the angular dislocation are valid only for angles $-\pi / 2 \leqq \beta \leqq \pi / 2$. Otherwise the slanting leg of the image dislocation cuts into the half space $x_{3} \geqq 0$ and gives an additional dislocation in the material.

This fact can be disregarded if angular dislocations are used to construct by superposition a closed polygonal loop. In such case, the undesired contributions of the angular image dislocations cancel automatically.

Nevertheless, an angular dislocation such that the slanting leg terminates at the surface is a valid problem in its own right and, furthermore, it is needed in the construction of open polygonal dislocations that start and terminate at the surface. As indicated in Fig. 2, however, the angular dislocation piercing the surface can be obtained by super-

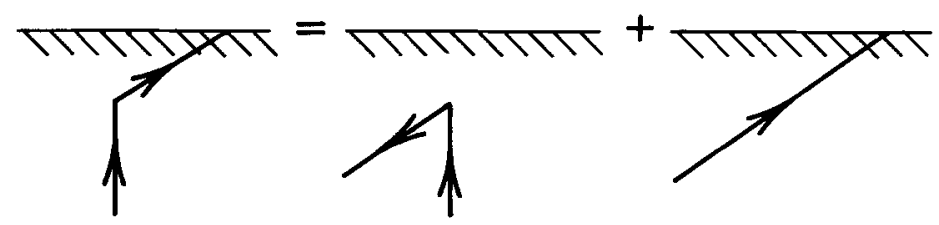

Figure 2. Angular dislocation piercing the surface of a half space.

posing an angular dislocation with $-\pi / 2<\beta<\pi / 2$ and a straight dislocation which terminates at the surface. The elastic fields for the latter have been given by Yoffe [7].

\section{Acknowledgement}

The reported results were obtained in the course of research supported by the National Science Foundation under the grant GK-35921.

\section{REFERENCES}

[1] Yoffe, E. H., The angular dislocation. Phil. Mag. 5, (1960) 161-175.

[2] Savage, J. C., Dislocations in Seismology. In Dislocation Theory, edited by F. R. N. Nabarro, Marcel Dekker, New York, in press

[3] Green, A. E., and W. Zerna, Theoretical Elasticity. Clarendon Press, Oxford 1968

[4] Dixon, D. A., Dislocation piercing a bimetallic interface. Dissertation, Northwestern University, 1966

[5] Comninou, M., Angular dislocation in a half space. Dissertation, Northwestern University, 1973

[6] Converse, G., private communication, 1972

[7] Yoffe, E. H., A dislocation at a free surface. Phil. Mag. 6(1961) 1147-1155 